\title{
Return to Play Following Musculoskeletal Injury
}

Editor

BRETT D. OWENS

\section{CLINICS IN \\ SPORTS MEDICINE}

www.sportsmed.theclinics.com

Consulting Editor

MARK D. MILLER

October 2016 • Volume 35 - Number 4 